(10\%) patients had HAS-BLED scores of more than 3 (high risk). Bleeding (cerebral, gastrointestinal, or hemoglobin $<100$ $\mathrm{g} / \mathrm{L})$ occurred in $32(12.3 \%)$ patients; 12 (37.5\%) of these patients had a HAS-BLED score of more than $3(\mathrm{p}=0.0001)$. TTR was not different between patients with or without bleeding $(64.1+19.4 \%$ versus $66.3+16.1 \%)$. On the other hand, mean HAS-BLED and ATRIA scores were significantly higher in patients who had bleeding. Using multivariate analysis, ATRIA score followed by HAS- BLED score was the best predictor of bleeding. Age, sex, and TTR as a measure of INR variability did not show a significant difference between the two groups.

Conclusion Similar to previous reports of patients with atrial fibrillation, ATRIA and HAS-BLED scores were the best predictors of bleeding in our cohort of patients with mechanical heart valves, with no independent contribution of TTR to estimation of bleeding risk.

\section{USING SIMULATION TO ASSESS COMPETENCY IN NEW NURSES}

Mohamed Noor Osman, Sami Alyateem. Nursing, Prince Mohammed Bin Abdulaziz Hospital Madinah

\subsection{6/bmjoq-2019-PSF.48}

Background Measuring competencies in nursing is critical to ensure safe and effective care of patients. Usually, new nurses struggle to cope with the demands of new roles and face difficulties in completing the required competencies on time. Being a clinical resource nurse based in the clinical areas and responsible for new nurses, I have observed that there is a gap in this area which may impact on patient safety. I work as a clinical resource nurse in Prince Mohammed Bin Abdulaziz Hospital in Madinah (PMBAH), Kingdom of Saudi Arabia. The focus of this paper is to assess the knowledge and confidence of newly hired nurses in PMBAH. Currently, new nurses are given 5 days of orientation in class and are then sent to clinical areas to start working under a senior nurse. The aim of this study was to assess the confidence and knowledge of new nurses hired by PMBAH.

Methods Simulation methodology was used to train new nurses in PMBAH. A 2-day training schedule was arranged with 2 hours for each competency session, with eight sessions in total. Each session included a short briefing about the scenario and introductory video followed by a demonstration by the simulation facilitator. The new nurse was then allowed to run the scenario with no interruption, followed by individual briefing directly to consolidate and transform the nurse's experience. Nurses were allowed to express their feelings in their own words. Pre and post self-assessment of participants' knowledge and confidence were later assessed.

Results There was a big difference in the pre- and post-assessment results; the majority of the nurses have shown increased knowledge and confidence after going through simulation sessions.

Conclusion We have identified that simulation positively impacts nurses' learning experiences by replicating the bedside setting in a mistake-free environment. Therefore, simulation sessions for new nurses are very helpful to raise knowledge, confidence, and the ultimate safe delivery of patient care. We can claim that simulation is an up-to-date and innovative learning strategy, especially when addressing direct patient care aspects including nursing skills and procedures. With the current increase in medicolegal cases, we can conclude that simulation is both a safer and economically viable way of training nurses. Increased confidence and knowledge using simulation during orientation has been shown to reduce stress levels in clinical areas.

\section{PRESCRIBER BEHAVIORS THAT COULD BE TARGETED FOR CHANGE: AN ANALYSIS OF BEHAVIORS DEMONSTRATED DURING THE PRESCRIBING PROCESS}

Mohammed Aseeri, Douha F Bannan, Aeshah AlAzmi, Mary P Tully. Pharmacy, King Abdulaziz Medical City

\subsection{6/bmjoq-2019-PSF.49}

Background The prescribing process for children with cancer is complex, and errors can occur at any step. As a result, many interventions have been used to reduce errors. However, few of them have been designed based on an understanding of the prescriber behavior that can lead to errors. In order to design effective behavior change interventions, it is first important to understand the prescribing process and identify prescriber behaviors that could be targeted for change.

Methods This study used two sequential phases. First, the prescribing process was observed and then described using the hierarchical task analysis (HTA) method. Second, prescriber tasks were analyzed using the behavior change wheel (BCW) approach to identify promising behaviors for change. These identified behaviors were prioritized based on information collected from focus groups with prescribers and chart review of errors made in the ward. The hospital's Institutional Review Board approval has been granted.

Results The HTA results showed that the prescribing process was complex and involved multiple tasks performed in varying orders. Applying the BCW identified 32 candidate behaviors for potentially reducing prescribing errors. However, after prioritizing these behaviors, only two emerged as promising candidate behaviors for intervention: writing drug indications at the time of prescription and using a predefined order sentence when ordering medications.

Conclusion Applying the HTA and BCW methods was helpful in identifying potential behaviors for change. Having identified promising behaviors, future work could explore what needs to change with respect to individuals and their work environments to achieve the desired change in these identified behaviors.

\section{SUCCESSFUL IMPROVEMENT IN THE QUALITY OF CLEANING AND DISINFECTION AT A SPECIALIZED TERTIARY CARE HOSPITAL IN RIYADH, SAUDI ARABIA}

Kassem Abou Yassine, Majid Alshamrani, Ayham Al Badawi, Nada Alrasheed, Mustafa Alhawtan, Basma Alaidan, Yahya Al Bakheet, Kholoud Alameer, Angela Caswell, Majid Malki, Aiman El-Saed. Infection Prevention and Control King Abdulaziz Medical City

\subsection{6/bmjoq-2019-PSF.50}

Background Environmental cleaning is critical for reducing the burden of healthcare-associated infections and multidrug-resistant organisms. The objective of the current study was to improve the quality of cleaning and disinfection done by housekeepers. 
Methods An interventional study was done between October 2018 and February 2019. The intervention included training of 130 housekeeping staff; redefining cleaning and disinfection responsibilities between housekeeping and nursing; adding a checklist for surfaces to be cleaned or disinfected; and emphasizing the inspector's auditing role. The intervention engaged relevant staff partners from infection control, housekeeping, nursing, and environmental services. The study outcome was the frequency of effective cleaning done by housekeepers. It was assessed by comparing the photos taken from specified room sites (pre-prepared by fluorescent gel) using black light before and after cleaning. Six highly touched areas in patient rooms were chosen. The study was divided into three phases: pre-intervention assessment (October 2018), intervention (November 2018 through January 2019), and post-intervention reassessment (February 2019).

Results A total of 27 rooms with 162 opportunities were assessed during the pre-intervention phase. The findings showed that only $39(24.1 \%)$ of the 162 opportunities were effectively cleaned. The frequencies of effective cleaning in different sites were: light switches $11.1 \%$, door knobs $25.9 \%$, water faucets $37 \%$, telephones $25.9 \%$, bed rails $14.8 \%$, and patient tables $29.6 \%$. A total of 33 rooms with 198 opportunities were assessed during the post-intervention phase. The findings showed that 116 (58.6\%) of the 198 opportunities were effectively cleaned. The frequencies of effective cleaning in different areas were: light switches $42.4 \%$, door knobs $84.8 \%$, water faucets $75.7 \%$, telephones $60.6 \%$, bed rails $54.5 \%$, and patient tables $63.6 \%$. The overall improvement in effective cleaning in different sites was $34.5 \% \quad(p<0.001)$, being highest for door knobs $(58.9 \%, \mathrm{p}<0.001)$ and lowest for light switches $(31.3 \%$, $\mathrm{p}=0.014)$.

Conclusion A multidisciplinary intervention including training and auditing of housekeepers was successful in significantly improving cleaning and disinfection at different sites in the patients' rooms. Frequent assessment and feedback may need to be continued until reaching an optimal level. Further studies are needed to evaluate the impact of improved cleaning on infection rates.

\section{MONITORING THE QUALITY INDICATORS OF BLOOD TRANSFUSION SERVICES AS A METHOD TO IMPROVE PATIENT SAFETY AT KING ABDULAZIZ UNIVERSITY HOSPITAL}

Salwa Hindawi, Tarek Elgemmezi, Seraj Alweail. Faculty of Medicine/Hematology and Blood Transfusion King Abdulaziz University - Jeddah - Saudi Arabia

\subsection{6/bmjoq-2019-PSF.51}

Background A quality indicator is measurable information gathered at the critical control points in a process or procedure for monitoring, assessment, and improvement. Quality monitoring is an important tool used to review blood transfusion practice and provide feedback on transfusion trends in blood transfusion services. Quality indicators can improve quality standards and support patient safety through setting priorities and process improvement. The aim of this study was to report 5 years' experience of monitoring the quality indicators at KAUH and to measure its impact on the blood transfusion practice as a tool in hemovigilance system implementation for patient safety.
Methods This was a retrospective study of data collected over the past 5 years (2013-2017) at KAUH, Jeddah, in which the quality indicators for certain parameters were analyzed and benchmarks were set for blood donor adverse reactions, transfusion reactions, fresh frozen plasma (FFP) indate wastage, and cross match to transfusion (CT) ratio. Data were forwarded to the Hospital Transfusion Committee (HTC) for review. Deviations were identified and corrective actions were taken. The outcomes were used to plan for improvement.

Results Among a total of 60,631 blood donors, 282 donor reactions were reported, resulting in a rate of $0.46 \%$, mostly in the form of mild dizziness. 285 adverse transfusion reactions were reported among 99,564 total blood transfusions, resulting in a rate of $0.28 \%$; most were allergic and febrile reactions. Monitoring of the adverse donor reactions showed a decreased incidence; however, the adverse transfusion reactions were under-reported. The FFP in-date wastage was 2205 among 22,590 requested FFP units, resulting in a high rate of 9.76\%. The CT ratio was 1.24. Safety improvements were implemented by a multidisciplinary quality improvement team to determine the critical control points and to address the factors contributing to high FFP wastage.

Conclusion The use of quality indicators as a tool for implementing a hemovigilance system can provide a better understanding of areas for improvement in the quality of the work and safety of patients. Establishing guidelines for appropriate clinical use of blood and proper communication between clinical transfusion staff and practitioners is expected to enhance these features along the blood transfusion chain. The use of a similar model in other institutions will facilitate the local benchmarking between hospitals, which is a feasible method to lower transfusion risk and cost and to improve quality outcomes.

\section{LAB QUALITY IMPROVEMENT PROJECT (MONITORING SEROLOGY REJECTION)}

Sarah Alharbi, Kaneez Zamir, Omar Qassas, Abdulaziz Johani, Abdulrahman Aboud Department of Pathology and Laboratory Medicine, Prince Mohammed Bin Abdul Aziz Hospital - Al Madinah

\subsection{6/bmjoq-2019-PSF.52}

Background This project was initiated because of the observation that serology rejection rates were increasing incrementally. The quality indicator displayed this; therefore, an intervention was necessary to reduce the amount of wastage of samples, patient recalls, supplies, and manpower. The aim of this project was to reduce the number of rejected samples, reduce recalls of patients and redraw of the samples, and to provide the best service to our customers.

Methods

- Using the quality indicators for the statistical measuring of the amount of serology samples rejected.

- PDCA (plan-do-check-act) cycle.

- Quality indicator:

- Numerator is serology rejected tests (lithium heparin and SST);

- Denominator is serology total tests done (lithium heparin and SST);

- International benchmark value $0.56 \%$;

- Baseline quality indicator was measured and then monitoring was done after implementation of interventions. 